\title{
Expression of BMP7 in cervical cancer and inhibition of epithelial-mesenchymal transition by BMP7 knockdown in HeLa cells
}

\author{
RUI SUN* , HONGWEI GUAN*, WEI LIU, JUNHUI LIANG, FEI WANG and CHANGZHONG LI \\ Department of Obstetrics and Gynecology, Shandong Provincial Hospital Affiliated to Shandong University, \\ Jinan, Shandong 250021, P.R. China
}

Received October 26, 2019; Accepted February 11, 2020

DOI: $10.3892 / \mathrm{ijmm} .2020 .4519$

\begin{abstract}
The aim of the present study was to investigate the expression of bone morphogenetic protein 7 (BMP7) in cervical cancer tissues, the effect of BMP7 on the proliferation, migration and epithelial-mesenchymal transition (EMT) of cervical cancer HeLa cells and the possible mechanism involved. Immunohistochemistry was used to stain the cervical cancer tissues and benign or precancerous lesions. Lentivirus containing BMP7 knockdown was transfected in HeLa cells and western blotting was performed to analyze BMP7 expression. At the same time, the influence of BMP7 knockdown on the expression of phosphorylated (p)-mothers against decapentaplegic homolog 1/5/9 and EMT-related markers [epithelial-cadherin, neural-cadherin, Vimentin, Snail and Slug] was detected. Cell Counting Kit-8 was used to detect cell proliferation. Transwell migration and invasion assays were performed to measure cell invasion and migration. The cell cycle was detected by flow cytometry. Compared with normal cervical epithelial and paracancerous cells, the positive rate of BMP7 expression in cervical cancer tissues was significantly increased. As compared with the control group, the expression of BMP7 was decreased in HeLa cells transfected with lentivirus. The knockdown of BMP7 in cervical cancer HeLa cells inhibited cell proliferation, migration and invasion, resulted in G1 cell cycle arrest and reversed the EMT process. In addition, the expression of $\mathrm{p}$-Smad1/5/9 was significantly decreased in HeLa cells with BMP7 knockdown. BMP7 is expected to be a possible target for the treatment of cervical cancer.
\end{abstract}

Correspondence to: Professor Changzhong Li, Department of Obstetrics and Gynecology, Shandong Provincial Hospital Affiliated to Shandong University, 324 Jingwuweisi Road, Jinan, Shandong 250021, P.R. China

E-mail: 15168888909@163.com

*Contributed equally

Key words: cervical cancer, BMP7, epithelial-mesenchymal transition, proliferation, invasion and migration

\section{Introduction}

Cervical cancer is the most common malignant tumor of the female reproductive tract, ranking 4th in incidence and mortality among female malignant tumors and first among reproductive tract malignancies worldwide (1). In China, the number of patients diagnosed with cervical cancer increases annually, with the age of patients decreasing (2). Cervical cancer seriously endangers the lives, health and safety of Chinese women. High-risk human papillomavirus (HPV) infection remains a major risk factor for cervical cancer (2). Clinically, radical surgery, supplemented radiotherapy or radiotherapy combined with cisplatin-based chemotherapy is the main treatment for cervical cancer. However, the most important characteristics of advanced cervical cancer are high recurrence rate, poor prognosis, local infiltration and distant metastasis. The development of effective adjuvant treatments is necessary.

Bone morphogenetic protein (BMP) is a member of the transforming growth factor- $\beta$ (TGF- $\beta$ ) superfamily. Over 20 subtypes of the BMP family have been identified in humans and played different roles in disease growth and progression (3). Among them, BMP7 is abnormally expressed in a variety of human tumors and is involved in regulating the proliferation, invasion and migration of cancer cells. BMP7 plays different roles in different tumors. In studies on ovarian cancer, BMP7 was found to be highly expressed in advanced ovarian cancer (4) and drug-resistant ovarian cancer cells (5). In digestive tract tumors, BMP7 expression was increased compared with in normal tissues and was associated with poor prognosis and the promotion of tumor invasion and metastasis $(6,7)$. In lung cancer, BMP7 could attenuate the activity and invasiveness of tumor cells, inhibit bone metastasis, and induce apoptosis and cell cycle arrest (8). In malignant melanoma, BMP7 can induce mesenchymal-epithelial transformation and inhibit the metastasis of cancer cells (9). Studies have found that BMP7 can inhibit epithelial-mesenchymal transition (EMT)-related genes and cell invasion $(10,11)$, inhibit telomerase, shorten telomeres, and induce the aging and apoptosis of breast cancer cells (12). BMP7 has also been found to increase the cell proliferation and migration potential in a model of metastatic breast cancer in the bone (13) and prostate cancer (14). 
When BMP7 binds to its receptor, it can lead to the intracellular phosphorylation of mothers against decapentaplegic homolog (smad)1/5/9 (also known as smad1/5/8) and further exerts its role in regulating cell proliferation (15). At present, studies on the association between the BMP7-Smad1/5/9 signaling pathway and cervical cancer are rare. The purpose of the present study was to verify the association between the BMP7-Smad1/5/9 signaling pathway and the occurrence, development and prognosis of cervical cancer, as well as to provide a new idea for the treatment of cervical cancer.

\section{Materials and methods}

Materials. Cervical cancer patients $(\mathrm{n}=100)$ undergoing surgery in the Department of Gynecology of the Provincial Hospital of Shandong University between January 2014 and November 2018 were included in the study, including 95 cases of squamous cell carcinoma (including 27 cases with corresponding paracancerous tissues), 4 cases of adenocarcinoma and 1 case of adenosquamous cell carcinoma (age range of all cases, 29-78 years). In the control group, 26 cervical tissue specimens were excised due to benign or precancerous lesions (age range, 30-72 years). All patients had no other complications. Human cervical cancer HeLa cells were purchased from the Cell Bank of the Chinese Academy of Sciences. Dulbecco's modified Eagle's medium (DMEM) was from Gibco (Thermo Fisher Scientific, Inc.), fetal bovine serum (FBS) from Biological Industries, BMP7 knockdown lentiviral vector (NM_001719) from GeneChem, Inc., Cell Counting Kit (CCK)-8 reagent from Dojindo Molecular Technologies, Inc. Transwell chambers and Matrigel were purchased from Corning Inc. The following antibodies were purchased: Rabbit anti-human polyclonal antibody to BMP7 (cat. no. ab56023; Abcam), rabbit anti-human polyclonal antibody to Smad1/5/9 (cat. no. ab66737; Abcam), rabbit anti-human monoclonal antibody to epithelial (E)-cadherin (cat. no. ab40772; Abcam), rabbit anti-human monoclonal antibody to neural (N)-cadherin (cat. no. ab76011; Abcam), rabbit anti-human monoclonal antibody to Vimentin (cat. no. ab92547; Abcam), rabbit anti-human polyclonal antibody to Snail + Slug (cat. no. ab85936; Abcam) and rabbit anti-human monoclonal antibody to GAPDH (cat.no. ab181602; Abcam). Rabbit anti-human polyclonal antibody to phosphorylated (p)-Smad1/5/9 (cat. no. 12656T; Cell Signaling Technology, Inc.) and horseradish peroxidase-labeled goat anti-rabbit secondary antibody (cat. no. ab205718; Abcam) were purchased. The bicinchoninic acid (BCA) protein concentration assay kit, SDS-PAGE, 3,3'-diaminobenzidine (DAB) coloring solution and other reagents were obtained from Beijing Solarbio Science \& Technology Co., Ltd.

Immunohistochemistry. Tissue samples were fixed at room temperature in formaldehyde ( 1-7 days) and embedded in paraffin, and then cut into $4-\mu \mathrm{m}$ sections, dewaxed with xylene, hydrated with $100-75 \%$ ethanol series, and repaired with sodium citrate ( $\mathrm{pH} \mathrm{6.0)}$ antigen in an autoclave $\left(100^{\circ} \mathrm{C}\right.$; $2 \mathrm{~min}$ ). Hydrogen peroxide was used to remove endogenous peroxidase. The goat serum (cat. no. SL038; Beijing Solarbio Science \& Technology Co., Ltd.) was used for blocking at $37^{\circ} \mathrm{C}$ for $30 \mathrm{~min}$ and BMP7 primary antibody (1:50) was then added at $4^{\circ} \mathrm{Covernight} \mathrm{for} \mathrm{incubation.} \mathrm{The} \mathrm{next} \mathrm{day,} \mathrm{the} \mathrm{goat} \mathrm{anti-rabbit}$ secondary antibody (1:200) was added for $30 \mathrm{~min}$ at $37^{\circ} \mathrm{C}$ and the slice was then stained with DAB at room temperature (observed until the staining was satisfactory) and hematoxylin for $2 \mathrm{~min}$ at room temperature. Images were captured under a cellSens light microscope (Olympus Corporation). Brown staining was considered to be BMP7-positive. A total of five fields of view were randomly selected from each slice, the degree of positive staining and the proportion of positive cells were recorded and scored (blind observation by two pathologists), and the positive rate was evaluated by the product of two items.

Cell culture. Human cervical cancer HeLa cells were inoculated in DMEM complete culture medium (containing $10 \%$ FBS, $1 \%$ penicillin and streptomycin) and cultured at $37^{\circ} \mathrm{C}$ in a $50-\mathrm{ml} / 1 \mathrm{CO}_{2}$ saturated humidity incubator, and the liquid was changed every 2 days. When the degree of cell fusion reached $80-90 \%$, cell passage was carried out using trypsin solution digestion and under strict aseptic conditions.

Lentivirus infection and grouping. The BMP7 knockdown lentivirus vector (NM_001719) was achieved by cloning short hairpin RNA (shRNA) using a self-inactivating lentivirus vector containing a CMV promoter-driven green fluorescent protein (GFP) reporter gene and a U6 promoter. The target sequence of BMP7 was 5'-GGATCTACAAGGACTACAT-3'. HeLa cells at the logarithmic growth stage were inoculated into 96-well plates at $5 \times 10^{4}$ cells $/ \mathrm{ml}, 100 \mu \mathrm{l}$ per well. The cells were transfected with lentivirus vector multiplicity of infection of 100. After $24 \mathrm{~h}$, normal medium was used to continue culture, passage and screening with puromycin. The transfection efficiency was monitored by ImageXpress Micro Confocal High-Content Imaging system and MetaXpress v6.2.3.733 High-content Image Acquisition and Analysis software (Molecular Devices, LLC). When the observed GFP expression reach 95\%, the next experiment was started. Cells were divided into the negative control (sh-NC) and experimental (sh-BMP7) groups.

Western blotting. sh-NC and sh-BMP7 cells at the logarithmic growth stage were used for experiments. Total cell protein was extracted by adding lysate [radioimmunoprecipitation assay lysis buffer (Beijing Solarbio Science \& Technology Co., Ltd.), phenylmethanesulfonyl fluoride and phosphatase inhibitor at a ratio of 100:1:1], and the protein concentration was determined using the BCA method. The protein ( $30 \mu \mathrm{g} / \mathrm{lane})$ was electrophoresed using a 10 or $12 \%$ SDS-PAGE gel, transferred using a polyvinylidene fluoride membrane and blocked with 5\% skimmed milk at room temperature for $1 \mathrm{~h}$. The primary antibodies were placed in a shaker at $4^{\circ} \mathrm{C}$ overnight for incubation. The primary antibody dilution concentration was as follows: Rabbit anti-BMP7 $(1: 2,000)$, rabbit anti-p-Smad1/5/9 (1:1,000), rabbit anti-E-cadherin $(1: 1,000)$, rabbit anti-N-cadherin $(1: 1,000)$, rabbit anti-vimentin $(1: 2,000)$, rabbit anti-Snail + Slug (1:2,000), rabbit anti-cyclinD1 $(1: 2,000)$ and rabbit anti-GAPDH $(1: 2,000)$. The membrane was then incubated with horseradish peroxidase-labeled goat anti-rabbit secondary antibody $(1: 5,000)$ for $1 \mathrm{~h}$ at room temperature and exposed to color using ECL reagent (Immobilon Western 
Chemiluminescent HRP substrate; EMD Millipore) and the Amersham Imager 600 (GE Healthcare).

Transwell assay. sh-NC and sh-BMP7 cells at the logarithmic growth stage were digested and collected, washed 3 times with PBS, and resuspended in serum-free medium to dilute the cells at $25 \times 10^{4}$ cells $/ \mathrm{ml}$.

In the cell migration assay, $200 \mu \mathrm{l}$ cell suspension was added in the upper well and medium containing $10 \%$ fetal bovine serum was added in the lower well. Following culture for $24 \mathrm{~h}$, the culture medium was discarded and washed 3 times with PBS. Cotton swabs were used to gently wipe off the cells on the upper well membrane. The lower well was fixed with $4 \%$ paraformaldehyde for $30 \mathrm{~min}$ at room temperature. Cells were washed 3 times with PBS again and stained with hematoxylin for $10 \mathrm{~min}$ at room temperature, rinse with tap water until blue. Following drying, the upper compartment membrane was cut off and placed on the slide for sealing. Images were captured under an inverted light microscope and counted using ImageJ v1.51 software (National Institutes of Health).

In the cell invasion assay, $90 \mu 1$ pre-diluted Matrigel (Matrigel: Serum-free medium, 1:7) was added to the upper well and deposited at $37^{\circ} \mathrm{C}$ overnight. The uncoagulated Matrigel was aspirated the next day. The cells were added and cultured for $48 \mathrm{~h}$. The other steps were the same as the migration experiment.

Cell cycle assay. sh-NC and sh-BMP7 cells were cultured and collected when the cell density reached $\sim 70 \%$ and cells were at the logarithmic growth stage. The cells were digested into single-cell suspension with trypsin, washed with PBS once and fixed with $75 \%$ ethanol solution (pre-cooled at $-20^{\circ} \mathrm{C}$ ) at $4^{\circ} \mathrm{C}$ overnight. After washing the cells, $2 \mu 1$ RNase (cat. no. R8021; Beijing Solarbio Science \& Technology Co., Ltd.) at a concentration of $1 \mathrm{mg} / \mathrm{ml}$ was added and the cells were bathed in water at $37^{\circ} \mathrm{C}$ for $40 \mathrm{~min}$. Next, $100 \mu \mathrm{l}$ propidium iodide (cat. no. G1021; Wuhan Servicebio Biotechnology Co., Ltd.) staining solution at a concentration of $100 \mu \mathrm{g} / \mathrm{ml}$ was added and cells were stained in the dark for $20 \mathrm{~min}$ at room temperature. Flow cytometry (CytoFLEX; Beckman Coulter, Inc.) was used to detect the cell cycle and Modfit Lt version 5.0 (Verity Software House, Inc.) to analyze cell cycle distribution.

Cell Counting Kit-8 cell proliferation assay. The assay was performed according to the manufacturer's protocol. Briefly, sh-NC and sh-BMP7 cells at the logarithmic growth stage were inoculated into 96 -well plates at $2 \times 10^{4}$ cells $/ \mathrm{ml}, 100 \mu \mathrm{l}$ per well, and cultured for 6, 24, 48 and $72 \mathrm{~h}$. The original medium was aspirated and $100 \mu \mathrm{l}$ medium containing $10 \%$ CCK-8 reagent was added into each well for incubation in a constant temperature incubator at $37^{\circ} \mathrm{C}$ for $1 \mathrm{~h}$. The absorbance of each well was measured at a wavelength of $450 \mathrm{~nm}$ and the absorbance of each hole was recorded to calculate the cell proliferation activity.

Statistical analysis. GraphPad Prism 7.0 software (GraphPad Software, Inc.) was used for statistical analysis. Pearson's $\chi^{2}$ test was used to compare differences in BMP7 expression levels between cervical cancer and normal cervical or paracancerous tissues. The t-test was used to compare the differences
Table I. Expression levels of BMP7 in cervical cancer tissues and paracancerous tissues (control).

\begin{tabular}{lccccc}
\hline Tissue & No. & Negative, $\mathrm{n}$ & Positive, $\mathrm{n}$ & $\chi^{2}$ & P-value \\
\hline Cancer & 27 & 3 & 24 & 35.9 & $<0.0001$ \\
Control & 27 & 25 & 2 & & \\
\hline
\end{tabular}

Table II. Expression levels of BMP7 in cervical cancer tissues and normal cervical epithelial tissues (control).

\begin{tabular}{lrcccc}
\hline Tissue & No. & Negative, $n$ & Positive, $n$ & $\chi^{2}$ & P-value \\
\hline Cancer & 100 & 22 & 78 & 44 & $<0.0001$ \\
Control & 26 & 24 & 2 & & \\
\hline
\end{tabular}

between the sh-BMP7 and sh-NC groups. Data are presented as the mean \pm standard deviation of at least three independent experiments. $\mathrm{P}<0.05$ was considered to indicate a statistically significant difference.

\section{Results}

BMP7 overexpression in cervical cancer tissues. According to the immunohistochemical results, BMP7 staining was mainly located in the cytoplasm and intercellular substance of the tissue. Cervical squamous cell carcinoma (Fig. 1A) and adenocarcinoma (Fig. 1C) tissues stained darker, paracancerous tissues (normal cervical tissue within $3 \mathrm{~cm}$ from the cancerous tissue with no cancer cell infiltration) (Fig. 1B) and normal glands (Fig. 1D) stained lighter. There was also low expression in normal cervical tissues (Fig. 1E). The expression of BMP7 in cervical cancer tissues (Fig. 1A and C) was statistically increased compared with in paracancerous tissues (Fig. 1B; Table I; P<0.05). BMP7 was highly expressed in cancer tissues (Fig. 1A and C), as compared with normal cervical epithelial tissues (Fig. 1D and E; Table II; P<0.05). When comparing the FIGO stage or pathological grade of cervical cancer tissues with the expression of BMP7, the difference was not significant.

Lentivirus transfection-induced BMP7 knockdown and western blotting verification. The two groups of HeLa cells were transfected by lentivirus and selected by puromycin for 5 days. The transfection efficiency was observed under fluorescent microscopy and the GFP expression was detected (Fig. 2A). The upper panels include light microscopic images and fluorescence microscopic images of sh-NC, the lower panels include light microscopic images and fluorescence microscopic images of sh-BMP7. The transfection efficiency was $>95 \%$. The western blotting results demonstrated that the BMP7 protein expression in the sh-BMP7 group was significantly decreased compared with in the sh-NC group. The expression of Smad1/5/9 did not change significantly in the sh-BMP7 group. In addition, the expression of $\mathrm{p}-\mathrm{Smad} 1 / 5 / 9$ was significantly decreased in the sh-BMP7 group (Fig. 2B-D). 

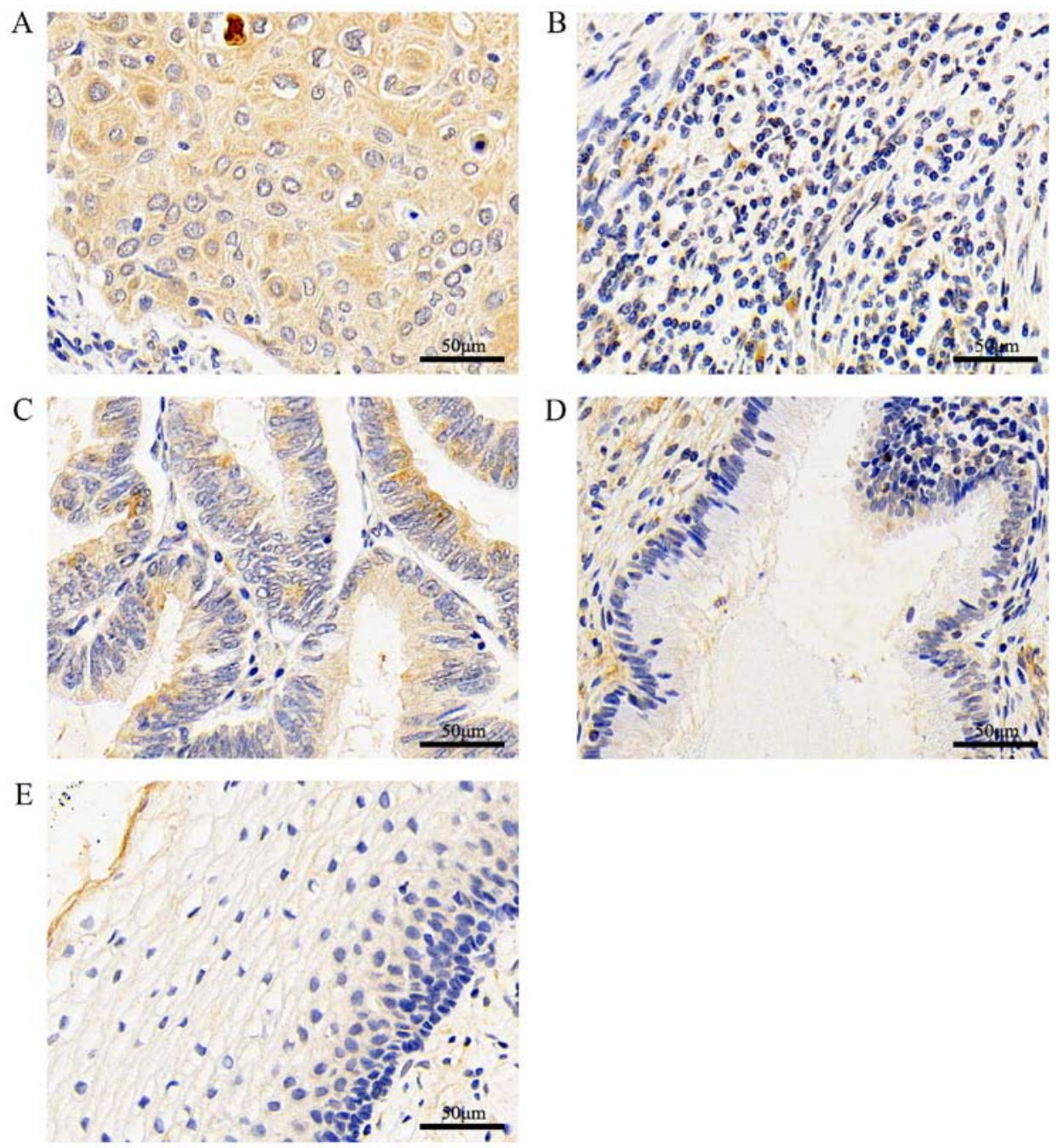

Figure 1. BMP7 expression in different types of cervical tissue. (A) Cervical squamous cell carcinoma tissue, (B) paracancerous tissue (normal cervical tissue within $3 \mathrm{~cm}$ from the cancerous tissue with no cancer cell infiltration), (C) cervical adenocarcinoma tissue, (D) normal cervical gland and (E) normal cervical squamous cell tissue (magnification, $\mathrm{x} 400$ ). The expression of BMP7 in cervical cancer tissues was higher than that in adjacent and normal cervical epithelial tissues. BMP7, bone morphogenetic protein 7.

EMT of HeLa cells is inhibited by BMP7 knockdown. Western blotting showed that, compared with the sh-NC group, the expression of EMT-related E-cadherin protein, which is an epithelial marker, was significantly increased in the sh-BMP group, while the expression of the $\mathrm{N}$-cadherin, vimentin, Snail and Slug proteins, which are mesenchymal markers, was decreased (Fig. 3), suggesting that EMT was inhibited in HeLa cells.

BMP7 knockdown can inhibit the invasion and migration of HeLa cells. In the Transwell invasion assay, the rate of Matrigel penetration in the sh-BMP7 group was decreased (Fig. 4A and C). The results of the Transwell migration assay showed that the cell migration rate was significantly decreased in the sh-BMP7 compared with in the sh-NC group (Fig. 4B and D). The invasion and migration ability of cells was reduced by BMP7 knockdown.

BMP7 knockdown inhibits the proliferation of HeLa cells. The cell cycle was detected by flow cytometry. Compared with the sh-NC group, the cell proliferation cycle was blocked in the
sh-BMP7 group. As shown in Fig. 5A and B, the proportion of sh-BMP7 cells at the G1 phase increased and the proportion of cells at the G2 phase decreased, compared with in the sh-NC group. These results suggested that the downregulation of BMP7 could cause G1 cell cycle arrest. Proliferation assay results showed that the cell proliferation rate of the sh-BMP7 group was decreased compared with the sh-NC group (Fig. 5C).

\section{Discussion}

Cassar et al (16) found that BMP7 could maintain telomerase activity, negatively regulate telomere maintenance and induce cervical tumor growth arrest in cervical cancer. However, the effect of BMP7 on EMT progression in cervical cancer cells has not been studied. The BMP-Smad signaling pathway plays a role in the development of a variety of tumors. As a member of the TGF- $\beta$ superfamily, BMP binds to type I and II serine/threonine kinase receptors, resulting in the phosphorylation of Smad, activation of downstream effectors, and control of cell proliferation, differentiation, metastasis, and apoptotic gene activation (17). BMP7 binds 
A
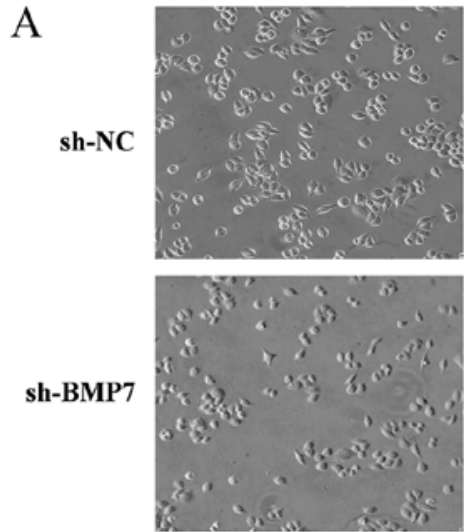

$\mathrm{C}$

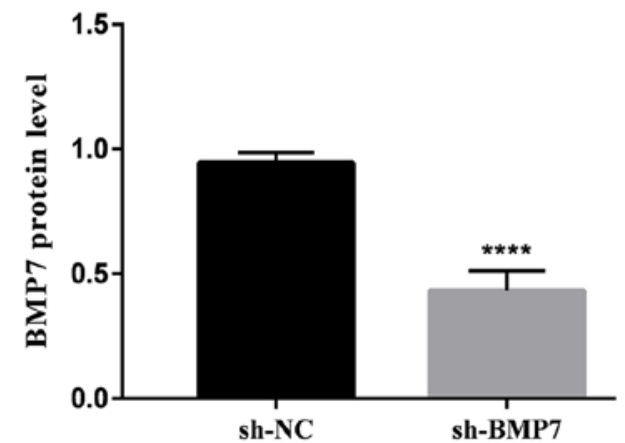

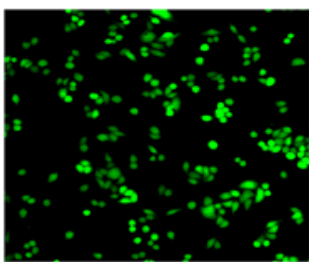

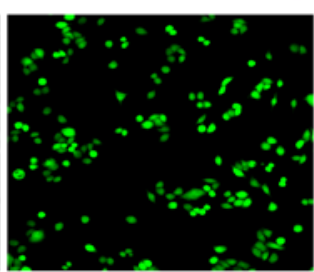

$\mathrm{D}$
B

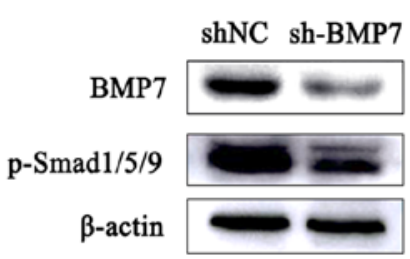

Smad1/5/9

GAPDH

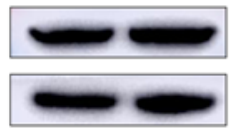

Figure 2. Expression of green fluorescent protein and BMP7, Smad1/5/9 and p-Smad1/5/9 protein in the sh-BMP7 and sh-NC groups of HeLa cells. (A) Green fluorescent protein GFP expression in sh-NC and sh-BMP7 groups of HeLa cells detected by fluorescent microscopy (magnification, x200). The upper panels are the light microscopic images and fluorescent microscopic images of sh- $\mathrm{NC}$, whereas the lower panels include light microscopic images and fluorescent microscopic images of sh-BMP7. (B) BMP7, Smad1/5/9 and p-Smad1/5/9 protein expression of the two groups, detected by western blotting. Densitometric analysis of (C) BMP7 and (D) p-Smad1/5/9 protein expression. " $\mathrm{P}<0.05$ and ${ }^{* * * * *} \mathrm{P}<0.0001, \mathrm{n} \geq 3 . \mathrm{BMP} 7$, bone morphogenetic protein 7; sh, short hairpin; $\mathrm{NC}$, negative control; smad, mothers against decapentaplegic homolog; $\mathrm{p}$, phosphorylated.

A

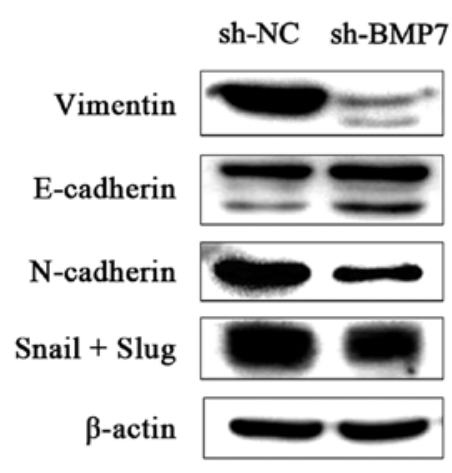

$\mathrm{B}$

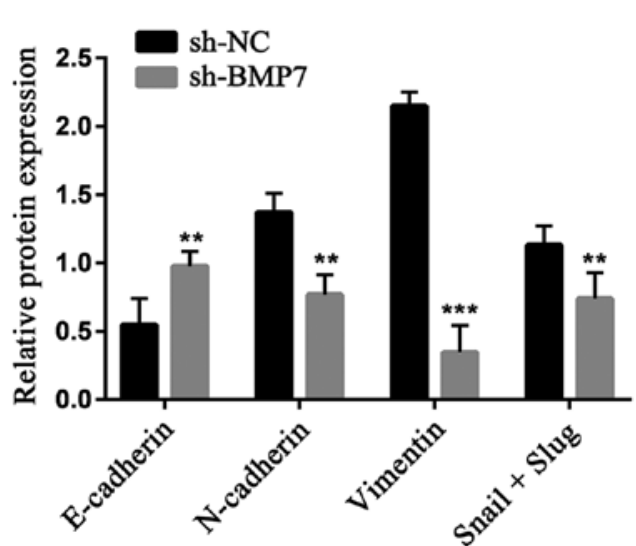

Figure 3. Expression of epithelial-mesenchymal transition-related proteins in the sh-BMP7 and sh-NC groups of HeLa cells. (A) Expression of epithelial-mesenchymal transition-related proteins in the sh-BMP7 and sh-NC groups of cells detected by western blotting. (B) Densitometric analysis of the protein expression. Following BMP7 knockdown, N-cadherin, Vimentin and Snail + Slug were downregulated, while E-cadherin was upregulated in HeLa cells. ${ }^{* *} \mathrm{P}<0.01$ and ${ }^{* * *} \mathrm{P}<0.001, \mathrm{n} \geq 3$. BMP7, bone morphogenetic protein 7; NC, negative control; E, epithelial; N, neural; sh-NC, short hairpin-negative control.

to ActR-II/IIB (type II) and ALK2/3/6 (type I) receptors, resulting in the phosphorylation of downstream effector Smad1/5/9 (R-Smad). Subsequently, p-Smad1/5/9 binds to Smad4, and enters and functions in the nucleus, acting on the promoter of target genes and initiating the transcription process, thus triggering specific biological effects (18). The Smad complex can affect the disease following nuclear entry by upregulating the inhibitors of differentiation (ID) (19). In previous years, studies have shown that ID regulate the differentiation of a variety of cells. In tumor cells, ID can promote angiogenesis and tumor cell invasion, inhibit apoptosis and promote cell immortality (20). In cervical cancer, elevated ID1 is associated with HPV infection and poor prognosis (21). 


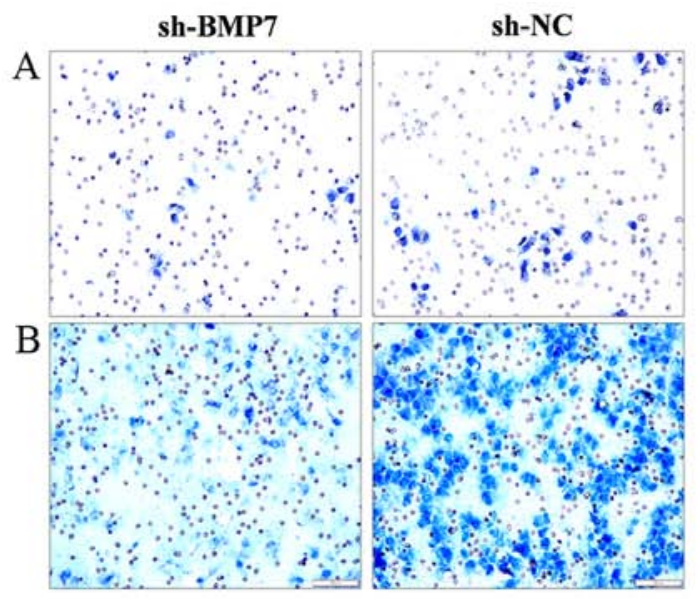

$\mathrm{C}$

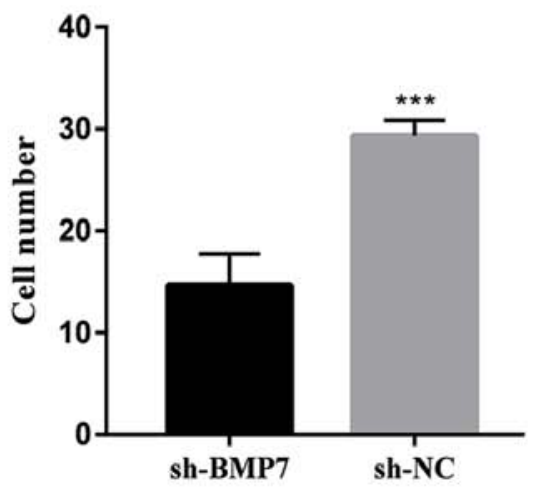

$\mathrm{D}$

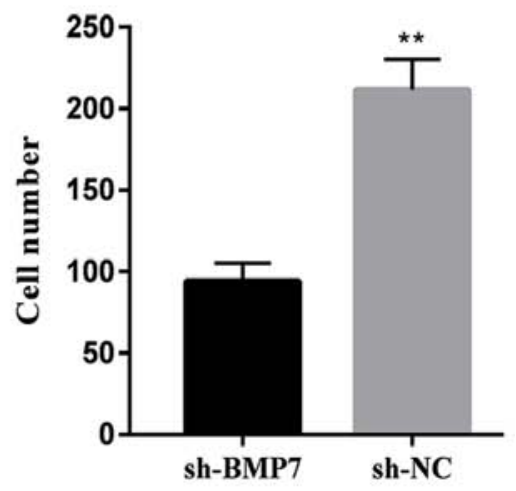

Figure 4. Cell invasion and migration ability of HeLa cells in the sh-BMP7 and sh-NC groups detected by Transwell assay. (A) Number of cells crossing through the Matrigel and well membrane following a $48 \mathrm{~h}$ incubation (magnification, x200). (B) Number of cells crossing through the membrane following a 24-h incubation (magnification, x200). The calculated value of the cell (C) invasion and (D) migration ability of HeLa cells following BMP7 knockdown. ${ }^{* *} \mathrm{P}<0.01$ and ${ }^{* * *} \mathrm{P}<0.001, \mathrm{n}=3$. BMP7, bone morphogenetic protein $7 ; \mathrm{NC}$, negative control.

A

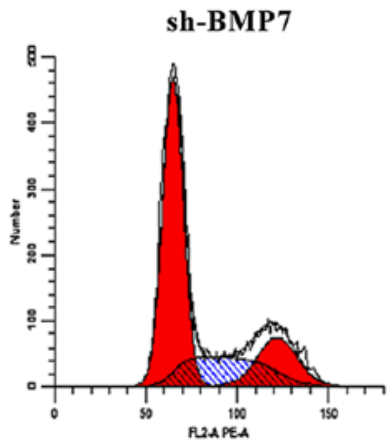

B

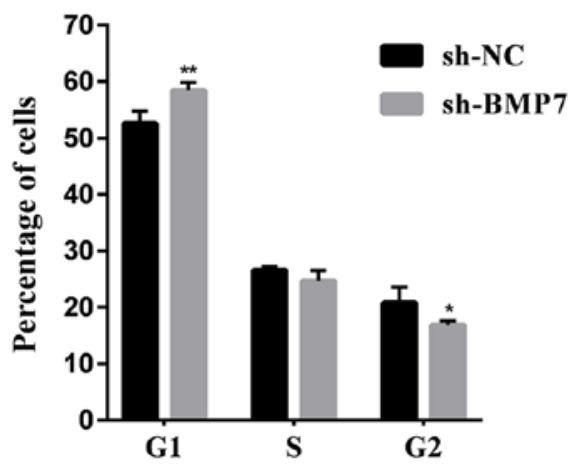

sh-NC

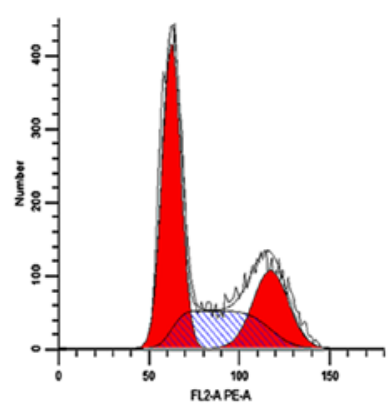

$\mathrm{C}$

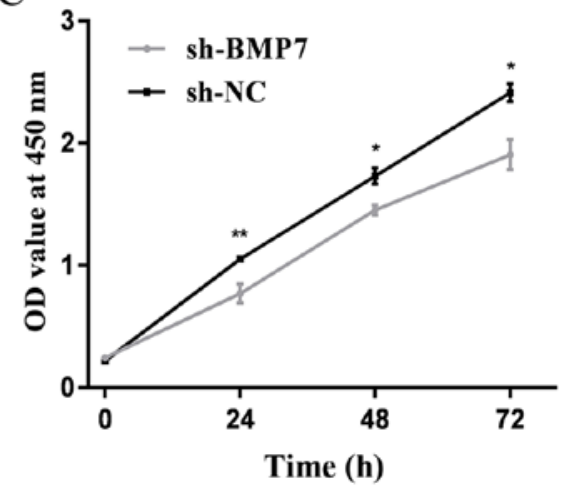

Figure 5. Cell cycle and proliferation analysis of the sh-BMP7 and sh-NC groups of HeLa cells. (A) Effect of BMP7 knockdown on the HeLa cell cycle. (B) The calculated value of the HeLa cell cycle following BMP7 knockdown. (C) Proliferation curve of HeLa cells in the sh-NC and sh-BMP7 groups. "P<0.05 and ${ }^{* *} \mathrm{P}<0.01, \mathrm{n}=3$. BMP7, bone morphogenetic protein 7; NC, negative control; sh, short hairpin; OD, optical density.

In the present study, based on immunohistochemical staining of cervical tissue in 125 patients, BMP7 was found to be highly expressed in cervical cancer tissues, as compared with normal cervical and paracancerous tissues. The present results are similar to those of a previous study (22). However, the difference in the expression intensity of BMP7 by pathological grade or clinical stage was not significant.

Based on the above conclusions, further cytological experiments were conducted. First, the expression of
BMP7 in HeLa cells was silenced by lentiviral transfection and the downstream factors were detected. It was found that the expression of $\mathrm{p}-\mathrm{Smad} 1 / 5 / 9$ downstream of the BMP $7 /$ Smad $1 / 5 / 9$ signaling pathway was decreased. The low expression of $\mathrm{p}$-Smad1/5/9 may further inhibit the proliferation and differentiation of cells. EMT research is an important part of tumor cell invasion and migration research. In tumor cells, EMT can generate circulating tumor cells and tumor stem cells, and improve resistance to anticancer drugs (23). Determining how to inhibit EMT in tumor cells is a major 
challenge for anti-tumor therapy. EMT is marked by the loss of the epithelial marker E-cadherin, upregulated expression of the interstitial cell markers $\mathrm{N}$-cadherin and vimentin, and zinc finger transcription factors Snail and Slug (24). EMT verification of HeLa cells was performed following transfection. EMT in HeLa cells was inhibited following BMP7 silencing, following which the expression of E-cadherin was increased and the expression of N-cadherin, vimentin, Snail and Slug proteins was decreased. In the Transwell migration and invasion experiments, the cell passage rate of the sh-BMP7 group was reduced. The invasion and migration ability of the sh-BMP7 group was weakened, which was the same as the expected results.

Muthukrishnan et al (25) showed that BMP7 can upregulate the $\mathrm{G} 1$ regulatory gene in renal tissue and Alarmo et al (26) showed that BMP7 silencing can lead to G1 growth arrest in breast cancer cells. In the present study, following BMP7 silencing, HeLa cells showed G1 phase growth arrest. Cell proliferation experiments showed that the proliferation rate of the sh-BMP7 group was decreased compared with the control group, which may be associated with G1 phase arrest.

In conclusion, BMP7 was highly expressed in cervical cancer. By knocking down BMP7 in cervical cancer HeLa cells, the expression of the downstream acting factor $\mathrm{p}-\mathrm{Smad} 1 / 5 / 9$ was reduced. BMP7 knockdown could also inhibit cervical cancer cell proliferation, invasion and migration, and reverse the EMT process. This effect may be caused by the inactivation of the BMP7-Smad1/5/9 signaling pathway. According to the present findings, BMP7 may be a potential therapeutic target for cervical cancer treatment.

\section{Acknowledgements}

Not applicable.

\section{Funding}

The present study was supported by the National Natural Science Foundation of China (grant no. 81671434).

\section{Availability of data and materials}

The analyzed data sets generated during the present study are available from the corresponding author on reasonable request.

\section{Authors' contributions}

RS wrote the manuscript. RS and HG interpreted the data and performed experiments. WL collected the data. JL and FW analyzed the data. CL designed and guided the study. All authors read and approval the final manuscript.

\section{Ethics approval and consent to participate}

All procedures performed in this study involving human participants were approved by the Biomedical Research Ethics Committee of Shandong Provincial Hospital (approval no. 2019-015). All patients provided written informed consent.

\section{Patient consent for publication}

Not applicable.

\section{Competing interests}

The authors declare that they have no competing interests.

\section{References}

1. Bray F, Ferlay J, Soerjomataram I, Siegel RL, Torre LA and Jemal A: Global cancer statistics 2018: GLOBOCAN estimates of incidence and mortality worldwide for 36 cancers in 185 countries. CA Cancer J Clin 68: 394-424, 2018.

2. Liu P: Big data evaluation of the clinical epidemiology of cervical cancer in mainland China. Chin J Pract Gynecol Obstet 34: 41-45, 2018

3. Bragdon B, Moseychuk O, Saldanha S, King D, Julian J and Nohe A: Bone morphogenetic proteins: A critical review. Cell Signal 23: 609-620, 2011.

4. Sunde JS, Donninger H, Wu K, Johnson ME, Pestell RG, Rose GS, Mok SC, Brady J, Bonome T and Birrer MJ: Expression profiling identifies altered expression of genes that contribute to the inhibition of transforming growth factor-beta signaling in ovarian cancer. Cancer Res 66: 8404-8412, 2006.

5. Cheng L, Lu W, Kulkarni B, Pejovic T, Yan X, Chiang JH, Hood L, Odunsi K and Lin B: Analysis of chemotherapy response programs in ovarian cancers by the next-generation sequencing technologies. Gynecol Oncol 117: 159-169, 2010.

6. Megumi K, Ishigami S, Uchikado Y, Kita Y, Okumura H, Matsumoto M, Uenosono Y, Arigami T, Kijima Y, Kitazono M et al: Clinicopathological significance of BMP7 expression in esophageal squamous cell carcinoma. Ann Surg Oncol 19: 2066-2071, 2012.

7. Aoki M, Ishigami S, Uenosono Y, Arigami T, Uchikado Y, Kita Y, Kurahara H, Matsumoto M, Ueno S and Natsugoe S: Expression of BMP-7 in human gastric cancer and its clinical significance. Br J Cancer 104: 714-718, 2011.

8. Shen W, Pang H, Xin B, Duan L, Liu L and Zhang H: Biological effects of BMP7 on small-cell lung cancer cells and its bone metastasis. Int J Oncol 53: 1354-1362, 2018.

9. Na YR, Seok SH, Kim DJ, Han JH, Kim TH, Jung H, Lee BH and Park JH: Bone morphogenetic protein 7 induces mesenchymal to-epithelial transition in melanoma cells, leading to inhibition of metastasis. Cancer Sci 100: 2218-2225, 2009.

10. Ying X, Sun Y and He P: Bone Morphogenetic Protein-7 inhibits EMT-associated genes in breast cancer. Cell Physiol Biochem 37: 1271-1278, 2015.

11. Kobayashi A, Okuda H, Xing F, Pandey PR, Watabe M, Hirota S, Pai SK, Liu W, Fukuda K, Chambers C, et al: Bone morphogenetic protein 7 in dormancy and metastasis of prostate cancer stem-like cells in bone. J Exp Med 208: 2641-2655, 2011.

12. Cassar L, Nicholls C, Pinto AR, Chen R, Wang L, Li H and Liu JP: TGF-beta receptor mediated telomerase inhibition, telomere shortening and breast cancer cell senescence. Protein Cell 8: 39-54, 2017.

13. Sakai H, Furihata M, Matsuda C, Takahashi M, Miyazaki H, Konakahara T, Imamura T and Okada T: Augmented autocrine bone morphogenic protein (BMP) 7 signaling increases the metastatic potential of mouse breast cancer cells. Clin Exp Metastasis 29: 327-338, 2012.

14. Lim M, Chuong CM and Roy-Burman P: PI3K, Erk signaling in BMP7-induced epithelial-mesenchymal transition (EMT) of PC-3 prostate cancer cells in 2- and 3-dimensional cultures. Horm Cancer 2: 298-309, 2011.

15. Boon MR, van der Horst G, van der Pluijm G, Tamsma JT, Smit JW and Rensen PC: Bone morphogenetic protein 7: A broad-spectrum growth factor with multiple target therapeutic potency. Cytokine Growth Factor Rev 22: 221-229, 2011.

16. Cassar L, Li H, Pinto AR, Nicholls C, Bayne S and Liu JP: Bone morphogenetic protein-7 inhibits telomerase activity, telomere maintenance, and cervical tumor growth. Cancer Res 68: 9157-9166, 2008.

17. Miyazono K, Kusanagi $\mathrm{K}$ and Inoue $\mathrm{H}$ : Divergence and convergence of TGF-beta/BMP signaling. J Cell Physiol 187: 265-276, 2001. 
18. Massagué J, Seoane J and Wotton D: Smad transcription factors. Genes Dev 19: 2783-2810, 2005.

19. Ruzinova MB and Benezra R: Id proteins in development, cell cycle and cancer. Trends Cell Biol 13: 410-418, 2003.

20. Roschger $C$ and Cabrele C: The Id-protein family in developmental and cancer-associated pathways. Cell Commun Signal 15: 7, 2017.

21. Xie L, Li J, Zhang Y, Liu B, Peng X, Lin Y, Xu W and $\mathrm{Hu}$ L: Inhibitors of differentiation-1 promotes nitrosopyrrolidine-induced transformation of HPV 16-immortalized cervical epithelial cell. Cancer Sci 105: 506-511, 2014.

22. Tang Z, Li C, Kang B, Gao G, Li C and Zhang Z: GEPIA: A web server for cancer and normal gene expression profiling and interactive analyses. Nucleic Acids Res 45 (W1): W98-W102, 2017.

23. Saitoh M: Involvement of partial EMT in cancer progression. J Biochem 164: 257-264, 2018.
24. Lamouille S, Xu J and Derynck R: Molecular mechanisms of epithelial-mesenchymal transition. Nat Rev Mol Cell Biol 15: 178-196, 2014.

25. Muthukrishnan SD, Yang X, Friesel R and Oxburgh L: Concurrent BMP7 and FGF9 signalling governs AP-1 function to promote self-renewal of nephron progenitor cells. Nat Commun 6: 10027, 2015.

26. Alarmo EL, Pärssinen J, Ketolainen JM, Savinainen K, Karhu R and Kallioniemi A: BMP7 influences proliferation, migration, and invasion of breast cancer cells. Cancer Lett 275: 35-43, 2009.

cc) (i) () $($ This work is licensed under a Creative Commons EY AC Attribution-NonCommercial-NoDerivatives 4.0 International (CC BY-NC-ND 4.0) License. 